\title{
INVESTIGACIONES
}

\section{La evaluación en educación física a través del "Game Perfomance Assessment Instrument" (GPAI)}

The assessment in physical education through the

"Game Perfomance Assessment Instrument" (GPAI)

A avaliação da educação fisica atravezo

"Game Perfomance Assessment Instrument" (GPAI)

\section{Javier Aguilar Sánchez, ${ }^{a}$ Ignacio Martín Tamayo, ${ }^{b}$ Luis Javier Chirosa Ríos ${ }^{c}$}

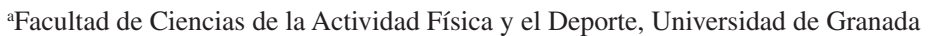 \\ Telf.: 696852766. Correo electrónico: jaguilarsanchez@ugr.es

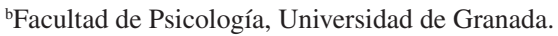 \\ Correo electrónico: imatin@ugr.es \\ ${ }^{\mathrm{c}}$ Facultad de Ciencias de la Actividad Física y el Deporte, Universidad de Granada \\ Correo electrónico: lchirosa@ugr.es
}

\begin{abstract}
RESUMEN
El uso de herramientas evaluativas del rendimiento deportivo que contribuyan a mejorar los procesos de enseñanza no está normalizado. Los objetivos de este artículo fueron obtener una visión actualizada del uso Game Perfomance Assessment Instrument (GPAI); y analizar su uso en la literatura científica para establecer nuevas líneas de investigación. Se utilizaron las bases de datos WEB OF SCIENCE, SPORTDISCUS, SCOPUS y PROQUEST, recogiendo artículos publicados entre enero de 1998 y mayo de 2015. De la muestra inicial de 582 investigaciones, 34 fueron analizadas tras aplicar los criterios de inclusión/exclusión. En 15, el GPAI ha servido para ver el progreso de los sujetos en distintos deportes tras periodos de enseñanza aprendizaje. Se ha usado principalmente en el ámbito educativo y con deportes de invasión. En conclusión, debido a las ventajas que presenta, su uso debería estar más extendido para dar solución al problema que la evaluación representa para muchos profesores.
\end{abstract}

Palabras clave: instrumento de evaluación, técnica, táctica, deportes.

\section{ABSTRACT}

The use of assessment tools for sports performance which contributes to improve the process of teaching is not formalized. The aims of this article were: to obtain an updated view of the Game Performance Assessment Instrument (GPAI) and, to analyze its use in scientific literature to establish new routes for investigation. The databases used were WEB OF SCIENCE, SPORTDISCUS, SCOPUS and PROQUEST, gathering articles from January of 1998 to May of 2015. From the initial sample of 582 studies, 34 were analyzed after applying the criteria of inclusion and exclusion. In 15, the GPAI has served to demonstrate the progress of the subjects in different sports after periods of teaching- learning. It has been used mainly in the education field and with invasion sports. In conclusion, due to the benefits that its presents, its use should be further extended to solve the problem that assessment represents to many teachers.

Key words: assessment instrument, technique, tactic, sports. 


\section{INTRODUCCIÓN}

La evaluación del rendimiento deportivo es un proceso muy complejo que debería abarcar todos los aspectos relacionados con el juego (Blomqvist, Vätinen y Luhtanen, 2005; Richard y Griffin, 2003). En el contexto educativo, la evaluación ha sido, durante los últimos 40 años, una de las cuestiones más problemáticas a las que los profesores de educación física han tenido que hacer frente (López et al., 2013). No se ha considerado como un mecanismo para mejorar el proceso de aprendizaje del alumno (Castelló y Cladellas, 2013) porque su finalidad ha consistido básicamente en asignar las calificaciones finales del proceso de enseñanza-aprendizaje (Blázquez, 2010; Hernández y Velázquez, 2004) sin tomar una decisión posterior en función del contexto y las personas afectadas. Diversos autores (Keating, 2003; López, 2011; Mitchell, Oslin y Griffin, 2006) han criticado el uso exclusivo de test físicos o de habilidad técnica en la evaluación del rendimiento en los deportes, prescindiendo de la evaluación de los aspectos tácticos. Estos test miden las habilidades fuera de contexto, sin reflejar la visión de conjunto del rendimiento del juego porque no representan la capacidad del estudiante jugador de ejecutar dichas habilidades en el momento y lugar adecuados (Oslin, Mitchell y Griffin, 1998).

En los últimos años, han surgido nuevas metodologías de enseñanza e instrumentos de evaluación que pretenden mejorar el proceso de enseñanza-aprendizaje. La metodología Teaching Games for Understanding (TGfU) (Bunker y Thorpe, 1982) trata de hacer más participes a los alumnos de su propio aprendizaje. Entre los instrumentos de evaluación, el GPAI, seguido del Team Sport Assessment Perfomance (TSAP), han sido los más utilizados para valorar la táctica en el ámbito educativo (Arias y Castejón, 2012). Dentro de las metodologías de evaluación presentadas por Godbout (1990), los dos instrumentos se encontrarían dentro de las estrategias cuantitativas, concretamente tendrían una posición intermedia entre los estadísticos derivados de la competición y los test estandarizados, en función de la situación (artificial o natural) en que se apliquen.

Oslin et al. (1998) diseñaron el GPAI para evaluar la toma de decisiones y el rendimiento de los jugadores. El instrumento es un sistema multidimensional que tiene como objetivo evaluar los comportamientos que se dan durante el juego para demostrar los conocimientos tácticos de los jugadores, así como la habilidad de los mismos para resolver problemas tácticos seleccionando y aplicando la técnica apropiada. El GPAI analiza los componentes individuales del juego (toma de decisiones, ejecución técnica, apoyo, etc.) y el rendimiento global en el juego (participación en el juego y rendimiento). Aunque todos los componentes están relacionados con el rendimiento en el juego, no se aplican a todos los deportes, y pueden simplificarse en función del objeto de estudio. Los comportamientos de los jugadores son registrados en hojas de observación y los componentes individuales del juego son calculados tras la aplicación de unas fórmulas propuestas por los autores.

Gréhaigne, Godbout y Bouthier (1997) desarrollaron un procedimiento de evaluación (TSAP) consistente en observar a un jugador durante un partido, registrando varios comportamientos que servirán para calcular el índice de eficiencia y el volumen de juego. Uno de los inconvenientes de este instrumento podría ser la ausencia de medida de las acciones en las que los sujetos no tienen la posesión del balón. Tampoco se miden las diferentes acciones defensivas, tan solo las recuperaciones de balón, por lo que el instrumento presenta ciertas limitaciones a la hora de evaluar el aprendizaje deportivo. 
Hasta donde se ha podido constatar en la literatura científica, en ningún estudio se ha analizado exclusivamente el uso del GPAI como herramienta evaluativa. En estudios precedentes (Arias y Castejón, 2012, 2014) la búsqueda se limitó hasta Enero del 2010 y se incluyó en el análisis el TSAP (Arias y Castejón, 2014), o el análisis del TSAP y otros instrumentos menos conocidos tras la aplicación de procesos de enseñanza-aprendizaje (Arias y Castejón, 2012). La importancia del tema radica en que el uso de este instrumento aún no es frecuente (Arias y Castejón, 2014; MacPhail, Kirk y Griffin, 2008) por lo que muchos profesores entrenadores no evalúan el rendimiento del juego o la táctica tras la enseñanza de cualquier deporte y no obtienen datos objetivos del rendimiento de los jugadores. Por tanto, se considera necesario esta investigación para realizar un análisis más amplio y actual de la producción científica acerca del uso del GPAI y su posible extensión al ámbito educativo extraescolar. Los objetivos de la presente investigación son los siguientes: obtener una visión actualizada del uso del GPAI en diferentes contextos, y analizar su uso en la literatura científica para establecer líneas de investigación relacionadas con el instrumento.

\section{METODOLOGÍA}

\subsection{ESTRATEGIAS PARA LA BÚSQUEDA BIBLIOGRÁFICA}

En la realización de esta investigación se han tenido en cuenta las consideraciones de algunos autores (Benito et al., 2007; González y Balaguer, 2007; Moher et al., 2009) y las propuestas por Preferred Reporting Items for Systematic reviews and Meta-Analyses (PRISMA) aplicables a las revisiones sistemáticas. La pregunta que plantea el problema al que se pretende dar respuesta es: cómo ha sido utilizado el GPAI hasta la actualidad.

Se revisaron artículos originales en inglés, español y portugués en las bases de datos WEB OF SCIENCE, SPORTDISCUS, SCOPUS y PROQUEST. La fecha de búsqueda fue el 30 de abril de 2015 y la acotación temporal entre enero de 1998 hasta la actualidad. La palabra clave introducida en los buscadores de las bases de datos fue "GPAI". La búsqueda fue realizada sin marcar ninguna opción en búsqueda avanzada, el motivo es porque marcando otras opciones (por ejemplo "title" o "título") disminuía de manera drástica el número de documentos, dejando de aparecer documentos considerados interesantes para la revisión.

\subsection{CRITERIOS DE INCLUSIÓN Y EXCLUSIÓN}

Tres especialistas participaron en la selección de investigaciones. Todos ellos tenían una amplia experiencia tanto en el ámbito educativo como en el alto rendimiento en diferentes deportes, disponiendo todos ellos de la máxima titulación federativa. Los criterios de selección de artículos se establecieron llegando a un consenso entre los investigadores. Se incluyeron: a) investigaciones descriptivas o experimentales en las que el GPAI fue utilizado; b) tesis doctorales. Se excluyeron del análisis: a) comunicaciones en congresos y póster; b) las tesis doctorales en las que el autor ya tenía otro/s artículos científicos publicados del mismo tema; c) artículos o capítulos de libros explicativos del GPAI; d) investigaciones publicadas en idiomas diferentes al español, inglés o portugués. 
Tras la selección de las investigaciones se procedió a un análisis más minucioso. Los resultados obtenidos fueron tabulados en una plantilla dividida en categorías para facilitar su análisis. En las categorías se incluían el/los deporte/s, los objetivos del estudio, la muestra, las diferentes variables, otros instrumentos utilizados y las principales conclusiones de cada artículo. Las investigaciones fueron analizadas teniendo en cuenta todas estas categorías.

Debido a la diversidad de métodos y de las variables medidas en los estudios, no fue posible utilizar escalas predefinidas y validadas para la evaluación de la cualidad metodológica de los artículos. Por eso, los protocolos de análisis utilizados y los resultados de algunos de los trabajos más interesantes fueron evaluados y discutidos de forma cualitativa.

\subsection{CARACTERIZACIÓN DE LA MUESTRA}

La muestra total inicial tras la búsqueda en las bases de datos anteriormente mencionadas fue de 582 investigaciones. Tras una primera lectura de las investigaciones y aplicación de los criterios de inclusión exclusión se seleccionó un total de 84 artículos. Tras una segunda lectura más exhaustiva, se seleccionaron, finalmente, 34 artículos como relevantes. Las diferencias de acuerdo entre los revisores fueron resueltas mediante discusión, siguiendo los criterios de inclusión exclusión predeterminados.

Figura 1. Diagrama de selección de referencias para la revisión sistemática

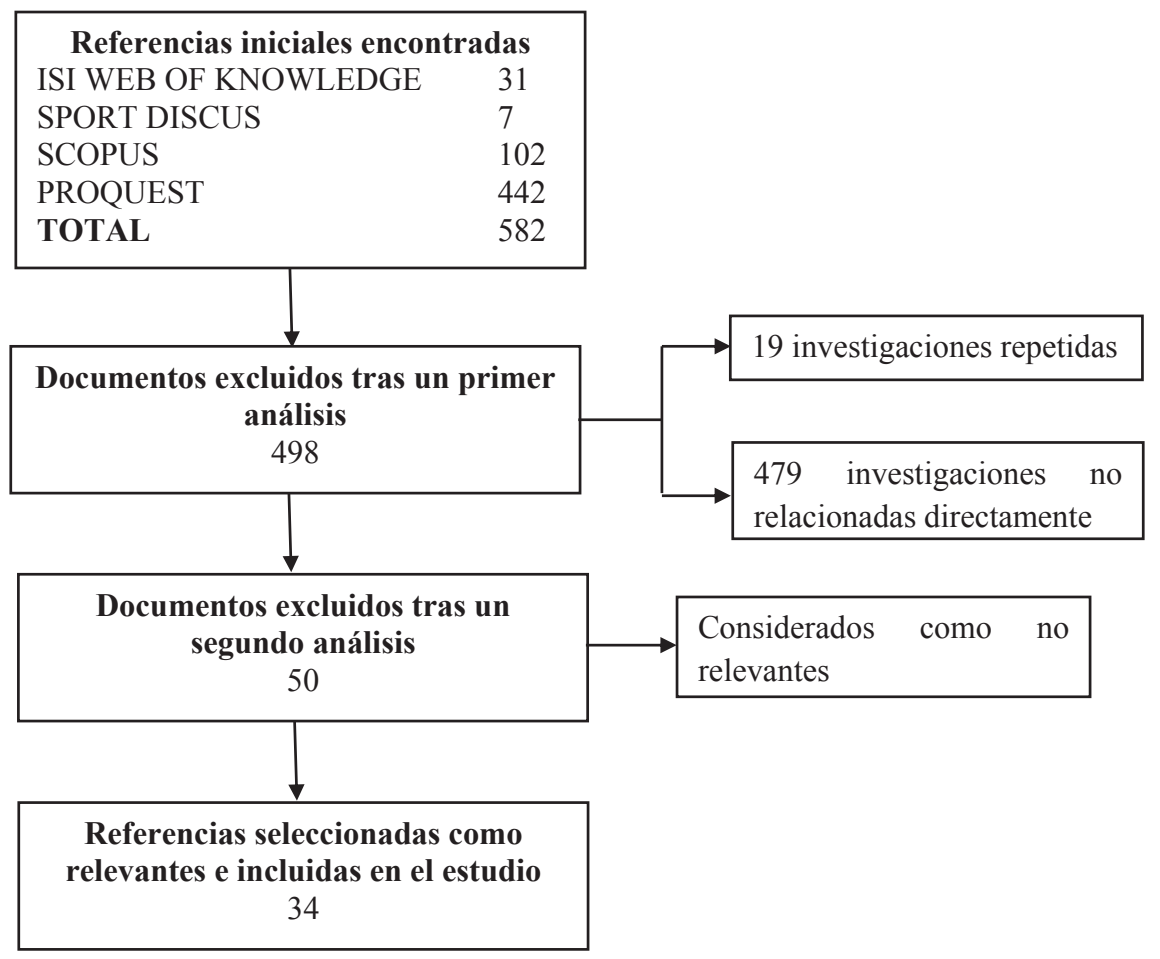




\section{RESULTADOS}

En los últimos años han sido numerosos los estudios en los que se ha utilizado el GPAI como herramienta evaluativa del rendimiento en el juego. De las 34 investigaciones analizadas, en 15 el GPAI ha servido para medir el progreso de los sujetos en distintos deportes tras periodos de enseñanza-aprendizaje basados en distintas metodologías. 10 investigaciones han seguido la metodología TGfU y derivadas, mientras que cinco investigaciones han seguido otras metodologías (ver Tabla 1). Cuatro investigaciones han valorado el GPAI por parte de profesores y dos investigaciones han definido de manera minuciosa las categorías del GPAI. En cinco investigaciones se han tenido en cuenta las consideraciones iniciales del GPAI para diseñar otros instrumentos. Ocho investigaciones han utilizado el GPAI con otros fines de interés.

Tabla 1. Estudios en los que se ha utilizado el GPAI en un proceso de enseñanza-aprendizaje

\begin{tabular}{|c|c|c|c|c|c|}
\hline Autores & $A \tilde{n} o$ & Objetivo & Muestra & $\begin{array}{l}\text { Proceso de } \\
\text { enseñanza- } \\
\text { aprendizaje }\end{array}$ & $\begin{array}{c}\text { Deporte } \\
\text { /juego }\end{array}$ \\
\hline Alanzi & 2013 & $\begin{array}{l}\text { Investigar los efectos de TGfU } \\
\text { sobre el aprendizaje y disfrute } \\
\text { de los alumnos en comparación } \\
\text { con otra metodología más tradi- } \\
\text { cional. }\end{array}$ & $\begin{array}{l}60 \text { estudiantes } \\
\text { (14-15 años) }\end{array}$ & $\begin{array}{c}T G f U \\
(20 \text { sesiones } \\
\text { de } 45 \text { minutos })\end{array}$ & fútbol \\
\hline $\begin{array}{l}\text { Balakrishnan, } \\
\text { Rengasamy } \\
\text { y Aman }\end{array}$ & 2011 & $\begin{array}{l}\text { Investigar los efectos de } T G f U \\
\text { sobre el aprendizaje cognitivo } \\
\text { de los estudiantes en compara- } \\
\text { ción con otra metodología más } \\
\text { tradicional. }\end{array}$ & $\begin{array}{l}72 \text { estudiantes } \\
\text { (10 años) }\end{array}$ & $\begin{array}{c}T G f U \\
\text { (4 semanas) }\end{array}$ & balonmano \\
\hline Bohler & 2011 & $\begin{array}{l}\text { Investigar los efectos de Tactical } \\
\text { Games Model sobre el aprendi- } \\
\text { zaje de la táctica, toma de deci- } \\
\text { siones y la transferencia. }\end{array}$ & $\begin{array}{l}18 \text { estudiantes } \\
\text { (10-11 años) }\end{array}$ & $\begin{array}{c}\text { Tactical } \\
\text { Games Model } \\
(20 \text { sesiones } \\
\text { de } 50 \text { minutos })\end{array}$ & $\begin{array}{l}\text { vóleibol, } \\
\text { bádminton, } \\
\text { pickleball }\end{array}$ \\
\hline Carpenter & 2010 & $\begin{array}{l}\text { Examinar la motivación para } \\
\text { estudiar el aprendizaje de los } \\
\text { estudiantes en distintas situa- } \\
\text { ciones deportivas. }\end{array}$ & $\begin{array}{l}15 \text { estudiantes } \\
\text { (11-12 años) }\end{array}$ & $\begin{array}{c}\text { Tactical } \\
\text { Games Model } \\
\text { (8 sesiones de } \\
40 \text { minutos) }\end{array}$ & $\begin{array}{l}\text { ultimate } \\
\text { frisbee }\end{array}$ \\
\hline $\begin{array}{l}\text { Chatzopoulos, } \\
\text { Drakou, } \\
\text { Kotzamanidou } \\
\text { y Tsorbatzoudis }\end{array}$ & 2006 & $\begin{array}{l}\text { Investigar los efectos de Tacti- } \\
\text { cal Games Model sobre el } \\
\text { aprendizaje y motivación de los } \\
\text { alumnos en comparación con } \\
\text { otra metodología más tradicio- } \\
\text { nal. }\end{array}$ & $\begin{array}{l}37 \text { estudiantes } \\
\text { (12-13 años) }\end{array}$ & $\begin{array}{c}\text { Tactical } \\
\text { Games Model } \\
(15 \text { sesiones } \\
\text { de } 45 \text { minutos })\end{array}$ & fútbol \\
\hline $\begin{array}{l}\text { Chatzopoulos, } \\
\text { Tsormbatzoudis } \\
\text { y Drakou }\end{array}$ & 2006 & $\begin{array}{l}\text { Investigar los efectos de Tacti- } \\
\text { cal Games Model sobre el } \\
\text { aprendizaje y motivación de los } \\
\text { alumnos en comparación con } \\
\text { otra metodología más tradicio- } \\
\text { nal. }\end{array}$ & $\begin{array}{l}101 \text { estudiantes } \\
\text { (12-13 años) }\end{array}$ & $\begin{array}{c}\text { Tactical } \\
\text { Games Model } \\
(15 \text { sesiones } \\
\text { de } 45 \text { minutos })\end{array}$ & fútbol \\
\hline $\begin{array}{l}\text { Harvey, Cushion, } \\
\text { Wegis y Massa- } \\
\text { Gonzalez }\end{array}$ & 2010 & $\begin{array}{l}\text { Investigar los efectos de } T G f U \\
\text { sobre los comportamientos de- } \\
\text { fensivos de los estudiantes. }\end{array}$ & $\begin{array}{c}34 \text { estudiantes } \\
\text { (14-18 años) }\end{array}$ & $\begin{array}{c}\text { Game Sense } \\
\text { (8 sesiones de } \\
45-60 \\
\text { minutos) }\end{array}$ & fútbol \\
\hline
\end{tabular}




\begin{tabular}{|c|c|c|c|c|c|}
\hline $\begin{array}{l}\text { Hastie, } \\
\text { Sinelnikov y } \\
\text { Guarino }\end{array}$ & 2009 & $\begin{array}{l}\text { Investigar los efectos de Sport } \\
\text { Education sobre el aprendizaje } \\
\text { de los estudiantes. }\end{array}$ & $\begin{array}{l}41 \text { estudiantes } \\
\text { (13-14 años) }\end{array}$ & \begin{tabular}{|c|} 
Sport \\
Education \\
$(18$ sesiones \\
de 45 minutos $)$
\end{tabular} & bádminton \\
\hline $\begin{array}{l}\text { MacPhail, Kirk y } \\
\text { Griffin }\end{array}$ & 2008 & $\begin{array}{l}\text { Investigar el aprendizaje de los } \\
\text { estudiantes con la metodología } \\
\text { Tactical Games Model. }\end{array}$ & $\begin{array}{l}29 \text { estudiantes } \\
\text { (9-10 años) }\end{array}$ & \begin{tabular}{|c|} 
Tactical \\
Games Model \\
(12 sesiones \\
de 40 minutos $)$
\end{tabular} & $\begin{array}{l}\text { juegos de } \\
\text { invasión }\end{array}$ \\
\hline $\begin{array}{l}\text { Mesquita, Farias } \\
\text { y Hastie }\end{array}$ & 2012 & $\begin{array}{l}\text { Investigar los efectos de Sport } \\
\text { Education-Invasion Games } \\
\text { Competence Model sobre el } \\
\text { aprendizaje de los estudiantes. }\end{array}$ & $\begin{array}{l}26 \text { estudiantes } \\
\text { (10-12 años) }\end{array}$ & $\begin{array}{c}\text { Sport } \\
\text { Education- } \\
\text { Invasion } \\
\text { Games } \\
\text { Competence } \\
\text { Model } \\
\text { (22 sesiones) }\end{array}$ & fútbol \\
\hline $\begin{array}{l}\text { Mesquita, Graca, } \\
\text { Gomes y Cruz }\end{array}$ & 2005 & $\begin{array}{l}\text { Investigar los efectos de Step } \\
\text { Games Approach sobre el } \\
\text { aprendizaje de los estudiantes. }\end{array}$ & $\begin{array}{l}25 \text { estudiantes } \\
\text { (12-15 años) }\end{array}$ & $\begin{array}{l}\text { Step Games } \\
\text { Approach } \\
\text { (12 sesiones) }\end{array}$ & vóleibol \\
\hline Panton & 2006 & $\begin{array}{l}\text { Investigar los efectos de un pro- } \\
\text { grama pedagógico en la eficacia } \\
\text { de la enseñanza de futuros pro- } \\
\text { fesores. }\end{array}$ & $\begin{array}{l}8 \text { profesores en } \\
\text { prácticas }\end{array}$ & 14semanas & varios \\
\hline $\begin{array}{l}\text { Pritchard, } \\
\text { Hawkins, } \\
\text { Wiegand y } \\
\text { Metzler }\end{array}$ & 2008 & $\begin{array}{l}\text { Investigar los efectos de Sport } \\
\text { Education sobre el aprendizaje } \\
\text { y motivación de los alumnos en } \\
\text { comparación con otra metodo- } \\
\text { logía más tradicional. }\end{array}$ & $\begin{array}{l}47 \text { estudiantes } \\
\text { (14-15 años) }\end{array}$ & $\begin{array}{c}\text { Sport } \\
\text { Education } \\
(20 \text { sesiones } \\
\text { de } 50 \text { minutos })\end{array}$ & vóleibol \\
\hline Psotta y Martin & 2011 & $\begin{array}{l}\text { Investigar los efectos de dos } \\
\text { modelos técnico-tácticos dife- } \\
\text { rentes sobre el aprendizaje de } \\
\text { los estudiantes. }\end{array}$ & $\begin{array}{l}24 \text { estudiantes } \\
\text { universitarios } \\
(20-22 \text { años })\end{array}$ & \begin{tabular}{|c|} 
Modelo \\
técnico-táctico \\
$(10$ sesiones \\
de 90 minutos $)$
\end{tabular} & fútbol \\
\hline $\begin{array}{l}\text { Tallir, Lenoir, } \\
\text { Valcke y } \\
\text { Musch }\end{array}$ & 2007 & $\begin{array}{l}\text { Investigar los efectos de Inva- } \\
\text { sion Games Competence Model } \\
\text { sobre el aprendizaje de los alum- } \\
\text { nos en comparación con otra } \\
\text { metodología más tradicional. }\end{array}$ & $\begin{array}{l}\text { 26 estudiantes } \\
\text { (10-11 años) }\end{array}$ & $\begin{array}{c}\text { Invasion } \\
\text { Games } \\
\text { Competence } \\
\text { Model } \\
\text { (12 sesiones) }\end{array}$ & baloncesto \\
\hline
\end{tabular}

Nota: en todas las investigaciones hubo alguna mejora en el aprendizaje tras la aplicación de la metodología de enseñanza, excepto en el estudio de Alanzi (2013) donde alumnos de séptimo grado mejoraron significativamente su rendimiento con TGfU en comparación con la metodología tradicional. Para los alumnos de octavo grado fue al contrario.

Balakrishnan, Rengasamy y Aman (2011) aplicaron la metodología TGfU en estudiantes de primaria en balonmano tras periodos de enseñanza de 4 y 6 semanas, mejorando los aprendizajes de los alumnos. Alanzi (2013) comparó la metodología TGfU con otra más tradicional en fútbol en estudiantes de primaria, obteniendo como resultado que los alumnos de séptimo grado mejoraron significativamente su rendimiento con TGfU. Para los alumnos de octavo grado fue al contrario.

El GPAI también ha sido usado para ver el progreso de los sujetos tras la aplicación de metodologías de enseñanza derivadas de TGfU. En todos los estudios se encontraron mejoras en los aprendizajes de los alumnos en alguno de los aspectos medidos. En fútbol, Harvey et al. (2010) evaluaron la metodología Game Sense (Australian Sports Commission, 1997) y las directrices marcadas por Meztler (2000) en alumnos de entre 14 y 18 años. Mesquita et al. (2005) utilizaron una metodología derivada de TGfU durante 12 sesiones en vóleibol 
en sujetos de entre 12 y 15 años. La metodología Tactical Games Approach (Mitchell et al., 2006) ha sido también analizada en diversas investigaciones. Bohler (2011) experimentó mejoras en el rendimiento de alumnos de primaria tras 20 sesiones de aprendizaje en deportes de red (bádminton, vóleibol y pickeball). En deportes de invasión, concretamente con el ultimate, Carpenter (2010) impartió ocho sesiones de esta metodología en estudiantes de primaria. MacPhail et al. (2008) la emplearon en estudiantes de primaria en 12 sesiones en diferentes juegos. Chatzopoulos et al. (2006) y Chatzopoulos, Tsormbatzoudis y Drakou (2006) la compararon con otra metodología centrada más en la técnica en fútbol en estudiantes de entre 12 y 13 años. Tallir et al. (2007) contrastaron la metodología Invasión Games Compentence Model (Musch et al., 2002) con otra más tradicional en estudiantes de entre 10 y 11 años en baloncesto.

La metodología Sport Education (Siedentop, 1994) también ha sido evaluada con el GPAI. Hastie, Sinelnikov y Guarino (2009) la aplicaron a estudiantes de primaria en 18 sesiones de bádminton. Pritchard, Hawkins y Wiegand (2008) utilizaron esta metodología durante 20 sesiones en estudiantes de secundaria para compararla con una más tradicional en voleibol. Mesquita, Farias y Hastie (2012) impartieron un modelo intermedio entre Sport Education e Invasión Games Compentence Model en fútbol con alumnos de primaria mejorando, sobre todo, los comportamientos de los sujetos con bajo nivel de habilidad. Psotta y Martin (2011) examinaron en fútbol a estudiantes de entre 19 y 21 años para confrontar dos métodos que integraban la técnica y la táctica. Panton (2006) evalúo con el GPAI el progreso de los alumnos tras un programa pedagógico en tenis, vóleibol, fútbol, baloncesto y rugby.

Auld (2006) intentó ver la relación existente entre el conocimiento táctico y el rendimiento táctico en varios juegos. Mitchell y Oslin (1999) estudiaron la transferencia táctica entre deportes de red con el GPAI. A. Moreno et al. (2011) analizaron los efectos de un programa de supervisión reflexiva en jugadores cadetes de vóleibol. Whipp et al. (2015) comprobaron el rendimiento en fútbol tras un periodo de enseñanza llevado a cabo por parte de los alumnos. Mann (2012) estudió la relación entre el feedback administrado por el entrenador y el rendimiento en jugadores de vóleibol de entre 16 y 18 años. M. P. Moreno et al. (2011) con el GPAI y otros instrumentos investigaron el perfil cognitivo de jugadoras expertas, para orientar la detección, formación y optimización de jóvenes talentos deportivos. Casey y Dyson (2009) mediante la investigación-acción aplicaron una metodología con el uso del GPAI en estudiantes de 11 y 12 años. Perlman, Forrest y Pearson, (2012) examinaron mediante el GPAI el rendimiento adquirido tras jugar, en videojuegos, béisbol, tenis, golf y bolos.

Aparte de las vertientes investigativas anteriores, Kuehl-Kitchen (2005) evaluó la experiencia de profesores en prácticas que en sus clases usaban TGfU y GPAI en hockey. Concluyó una valoración positiva de los profesores hacia el GPAI como instrumento de evaluación del rendimiento durante las clases, pero también descubrió dificultades por parte de los profesores a la hora de interpretar los resultados obtenidos en el GPAI. En esta misma línea, Gubacs (2000) investigó a profesores en prácticas y la percepción de sus tutores en relación a la implementación de TGfU y GPAI en 8 semanas de clases de tenis. El rendimiento de los profesores también ha sido evaluado por Wright et al. (2005) en baloncesto, al comparar TGfU con otra metodología de entrenamiento basada en la técnica en futuros profesores de educación física. Los resultados revelan que el grupo entrenado bajo la metodología TGfU mejoró algunos índices. Conjuntamente mejoraron 
los resultados en un cuestionario para revelar sus percepciones acerca de cómo enseñar la táctica y estrategia. Roberts (2007) comprobó el rendimiento de profesores de educación física en varios juegos tácticos con el GPAI.

Por último, se han propuesto definiciones para cada categoría del GPAI en las diferentes categorías de deportes (Méndez, 2011a) y específicamente para los deportes de red (Hopper, 2003). En otras investigaciones se han creado instrumentos de evaluación teniendo en cuenta algunas consideraciones aportadas por el GPAI en fútbol (Da Costa et al., 2011; Otero, González y Calvo, 2012), baloncesto (Chen, Hendricks y Zhu, 2013; Folle et al., 2014) y vóleibol (Collet et al., 2011).

\section{DISCUSIÓN}

Los resultados obtenidos en el presente trabajo satisfacen los objetivos de la investigación y aportan datos de interés para el ámbito científico y educativo, puesto que se ha analizado de manera minuciosa el uso del GPAI hasta la actualidad.

En la mayoría de las investigaciones se ha utilizado el GPAI como instrumento de evaluación tras un periodo de enseñanza obteniendo mejoras al final del proceso en muchas de las variables medidas. De entre todas las investigaciones desarrolladas en el ámbito de la enseñanza, tan solo tres han sido realizadas en el contexto extraescolar o universitario. La mayoría de estas investigaciones ha evaluado deportes de invasión. Cuatro investigaciones han evaluado deportes de red.

El periodo de enseñanza se basa, en muchos estudios, en la aplicación de la metodología de enseñanza TGfU y derivadas (Balakrishnan et al., 2011; Bohler, 2011; Carpenter, 2010; Harvey et al., 2010; MacPhail et al., 2008; Mesquita et al., 2005) u otras (Hastie et al., 2009; Mesquita et al., 2012; Panton, 2006). Incluso en algunos estudios se comparan algunas de las metodologías anteriores con otras más tradicionales (Alanzi, 2013; Chatzopoulos et al., 2006; Chatzopoulos, Tsormbatzoudis y Drakou, 2006; Pritchard et al., 2008; Psotta y Martin, 2011; Tallir et al., 2007). Con el GPAI también se ha estudiado la relación existente entre el conocimiento táctico y el rendimiento táctico (Auld, 2006) y se ha comprobado la transferencia táctica entre diferentes deportes (Mitchell y Oslin, 1999). Igualmente se ha utilizado para ver los efectos de un programa de supervisión reflexiva (M. P. Moreno et al., 2011), para evaluar periodos de microenseñanza (Whipp et al., 2015), para conocer el perfil cognitivo de deportistas (Otero et al., 2012) e incluso para comprobar el rendimiento en el juego después de jugar con videojuegos (Perlman et al., 2012). En otros estudios se ha analizado la opinión de los profesores en el uso del GPAI (Gubacs, 2000; KuehlKitchen, 2005; Wright et al., 2005). También se han concretado las categorías del GPAI (Hopper, 2003; Méndez, 2011a) y se han creado instrumentos teniendo en cuenta algunas consideraciones del GPAI (Chen et al., 2013; Collet et al., 2011; Da Costa et al., 2011; Folle et al., 2014; Otero et al., 2012).

Entre las ventajas del GPAI se podrían destacar las siguientes: a) permite evaluar todos los componentes del rendimiento del juego; b) se puede adaptar a cada categoría de deporte y a los objetivos que se pretendan alcanzar con su uso; c) puede ofrecer información de interés para detectar los problemas de rendimiento en el juego y diseñar estrategias para corregirlos; d) puede facilitar la transferencia entre los principios de juego de diferentes categorías para hacer los aprendizajes más significativos; e) es una herramienta fiable y 
válida; y f) permite llevar a cabo diferentes tipos de evaluación (evaluación recíproca, autoevaluación, heteroevaluación). A pesar de las ventajas que presenta el instrumento, Memmert y Harvey (2008) pusieron de manifiesto algunos problemas de codificación y cálculo. Dos de estos problemas se podrían solucionar (1) creando nuevos índices que resulten de la suma de las conductas adecuadas eficaces [Índice de Conductas Adecuadas/ Eficaces (ICAE)]; (2) de la suma de las conductas inadecuadas/ineficaces [Índice de Conductas Inadecuadas/Ineficaces (ICII)]; (3) de la diferencia entre ambas [Índice de Diferencia entre Conductas (IDC)]; y (4) de la suma de ambas [Índice Global (IG)]. Así, al asemejar el IDC al Índice de Rendimiento en el Juego (IRJ) se soluciona el problema de no linealidad. Además el IDC y el IG se podrían comparar fácilmente al estar medidos en la misma escala, no pasaba lo mismo con el IRJ y el Índice de Participación en el Juego (IPJ). Ello se aprecia en la Figura 2.

Figura 2. Esquematización de soluciones a los problemas evidenciados por Mermmert y Harvey (2008)

$$
\begin{gathered}
I C A E=\sum_{c=1}^{n} C A E_{C} \\
I C I I=\sum_{d=1}^{m} C I I_{m} \\
I D C=I C A E-I C I I \\
I G=I C A E+I C I
\end{gathered}
$$

Otros problemas detectados en el uso del GPAI (Kuehl-Kitchen, 2005; Méndez, 2011b) se podrían solucionar resolviendo la falta de experiencia en su uso o se considera que estarían siempre presentes con la utilización de cualquier instrumento de evaluación. Alarcón et al. (2011) exponen que el GPAI no abarca todas las posibilidades que tiene el jugador de responder en cada situación. En este sentido se supone que en el ámbito educativo no es necesario evaluar todas las posibilidades del jugador y para el alto rendimiento se podrían crear nuevas categorías en función de los objetivos que se persigan.

En conclusión, debido a sus ventajas el GPAI ha sido utilizado principalmente en el ámbito escolar. Algunas investigaciones también lo han utilizado en el alto rendimiento y para crear otros instrumentos de evaluación. Aun así, la evaluación en educación física sigue siendo hoy día un problema para muchos profesores, por lo que se establece la necesidad de usar modelos alternativos de evaluación para ayudar a los estudiantes en sus aprendizajes y a los profesores a mejorar el proceso de enseñanza (López et al., 2013). Con el uso adecuado del instrumento se podría llegar a lo que Chaverrá (2014) denomina como racionalidad práctica, que permitiría reconocer la evaluación como un ejercicio crítico que revisa las interacciones que se van produciendo entre estudiante, profesor y conocimiento. El GPAI no se ha utilizado en una amplia variedad de juegos y deportes (Arias y Castejón, 
2012) a pesar de que algunos autores (Brown y Hopper, 2008; Griffin y Richard, 2003; Harvey, 2007; Light y Fawns, 2003; Siendetop, 2002) respaldan su uso como instrumento evaluativo del rendimiento global del juego. Por tanto, los investigadores deberían hacer un esfuerzo para acercar el GPAI a los profesores y entrenadores (Arias y Castejón, 2014) y así poder sistematizar y estandarizar el instrumento.

En futuras investigaciones podría ser interesante utilizar el GPAI para ver la posible transferencia táctica entre diferentes deportes o juegos, para comparar la eficacia de distintas metodologías basadas en TGfU y para aplicar modelos de evaluación recíproca entre alumnos. Asimismo, para facilitar la labor del docente es necesario definir concretamente las categorías del GPAI para cada tipo de deporte y la creación de un software estandarizado.

\section{REFERENCIAS BIBLIOGRÁFICAS}

Alanzi, W. K. (2013). Middle School students' game play performance and levels of enjoyment while engaged in two curricular models (Faculty of the Graduate School at Middle Tennessee State University). Murfreesboro, Estados Unidos.

Alarcón, F., Cárdenas, D., Miranda, M. T., Ureña, M., \& Piñar, M. I. (2011). Influencia de un programa de entrenamiento sobre la movilidad en baloncesto. Revista Internacional de Medicina y Ciencias de la Actividad Física y el Deporte, 11(44), 749-766.

Arias, J. L., \& Castejón, F. (2012). Review of the instruments most frequently employed to assess tactics in physical education and youth sports. Journal of Teaching in Physical Education, 31(4), 381-391.

Arias, J. L., \& Castejón, F. (2014). Using instruments for tactical assessment in physical education and extra-curricular sports. European Physical Education Review, 20(4), 525-535.

Auld, R. K. (2006). The relationship between tactical knowledge and tactical performance for varying levels of expertise (University of Rhode Island and Rhode Island College). Providence, Estados Unidos.

Australian Sports Commission. (1997). Games Sense: Developing thinking players. Camberra: Author.

Balakrishnan, M., Rengasamy, S., \& Aman, M. S. (2011). Effect of teaching games for understanding approach on students' cognitive learning outcome. World Academy of Science, Engineering and Technology, 77, 961-963.

Benito, P. J., Díaz, V., Calderón, F. J., Peinado, A. B., Martín, C., Álvarez, M., \& Pérez, J. (2007). La revisión bibliográfica sistemática en fisiología del ejercicio: recomendaciones prácticas. Revista Internacional de Ciencias del Deporte, 3(6), 1-11.

Blázquez, D. (2010). Evaluar en educación física (11ra ed.). Barcelona: INDE.

Blomqvist, M., Vätinen, T., \& Luhtanen, P. (2005). Assessment of secondary school students' decision-making and game-play ability in soccer. Physical Education and Sport Pedagogy, 10(2), 107-119.

Bohler, H. R. (2011). Fifth-grade students' tactical understanding, decision-making and transfer of knowledge in a tactical games model net/wall sampling unit (University of Massachusetts). Amherst, Estados Unidos.

Brown, S., \& Hopper, T. (2008). Can all students in PE get an 'A'? Game performance assessment by peers as a critical component of student learning. The PHE, 72(1), 14-21.

Bunker, D., \& Thorpe, R. (1982). A model for the teaching of games in secondary schools. Bulletin of Physical Education, 18(1), 5-8.

Carpenter, E. J. (2010). The tactical games model sport experience: An examination of student motivation and game performance during an ultimate frisbee unit (University of Massachusetts). 
Amherst, Estados Unidos.

Casey, A., \& Dyson, B. (2009). The implementation of models-based practice in physical education through action research. European Physical Education Review, 15(2), 175-199.

Castelló, A., \& Cladellas, R. (2013). La evaluación de la comprensión en el aprendizaje: El empleo de las TIC en el análisis de estructuras de conocimiento. Estudios Pedagógicos, 39(1), 41-57.

Chatzopoulos, D., Drakou, A., Kotzamanidou, M., \& Tsorbatzoudis, H. (2006). Girls' soccer performance and motivation: games Vs technique approach. Perceptual y Motor Skills, 103(2), 463-470.

Chatzopoulos, D., Tsormbatzoudis, H., \& Drakou, A. (2006). Combinations of technique and games approaches: Effects on game performance and motivation. Journal of Human Movement Studies, 50(3), 157-170.

Chaverra, B. (2014). Significados otorgados a la evaluación de la enseñanza y el aprendizaje. Interpretación a partir de un grupo de maestros de educación física. Estudios Pedagógicos, 40(2), 65-82.

Chen, W., Hendricks, K., \& Zhu, W. (2013). Development and validation of the basketball offensive game performance instrument. Journal of Teaching in Physical Education, 32(1), 100-109.

Collet, C., Do Nascimento, J. V., Ramos, V., \& Stefanello, M. F. (2011). Construction and validation of a technical-tactical performance evaluation instrument in volleyball. Revista Brasileira de Cineantropometria y Desempenho Humano, 13(1), 43-51.

Da Costa, I. T., Garganta, J., Greco, P. J., Mesquita, I., \& Maia, J. (2011). Sistema de avaliação táctica no Futebol (FUT-SAT): Desenvolvimento e validação preliminar. Motricidade, 7(1), 69-84.

Folle, A., Teixeira, R., Couto, M. L., Scmitt, J. L., Ramos, V., \& Vieira, J. (2014). Construção e validação preliminar de instrumento de avaliação do desempenho técnico-tático individual no basquetebol. Revista da Educação Física/UEM, 25(3), 405-418.

Godbout, P. (1990). Observational strategies for the rating of motor skill. Theoretical and practical implications. En M. Lirette, C. Paré, J. Dessureault, \& M. Pieron (Eds.), Physical education and coaching: Present state and outlook for the future (pp. 209-221). Québec: Presses de 1'Université du Québec á Trois- Riviéres.

González, J., \& Balaguer, A. (2007). Revisión sistemática y metanálisis (I): Conceptos básicos. Evidencias en Pediatría, 3(107), 1-10.

Gréhaigne, J.-F., Godbout, P., \& Bouthier, D. (1997). Performance assessment in team sports. Journal of Teaching in Physical Education, 16(4), 500-516.

Griffin, L. L., \& Richard, J. F. (2003). Using authentic assessment to improve students' net/wall game play. Teaching Elementary Physical Education, 14(2), 23-27.

Gubacs, K. D. (2000). Action research on a tactical approach to teaching a Pre-Service tennis class (University of Massachusetts). Amherst, Estados Unidos.

Harvey, S. (2007). Using a generic invasion game for assessment. Journal of Physical Education, Recreation y Dance, 78(4), 19-25, 48-50.

Harvey, S., Cushion, C. J., Wegis, H. M., \& Massa-Gonzalez, A. N. (2010). Teaching games for understanding in American high-school soccer: A quantitative data analysis using the Game Performance Assessment Instrument. Physical Education y Sport Pedagogy, 15(1), $29-54$.

Hastie, P. A., Sinelnikov, O. A., \& Guarino, J. (2009). The development of skill and tactical competencies during a season of badminton. European Journal of Sport Science, 9(3), 133-140.

Hernández, J., \& Velázquez, R. (2004). La evaluación en educación física. Investigación y práctica en el ámbito escolar. Barcelona: Graó.

Hopper, T. (2003). Four Rs for tactical awareness: Applying Game Perfomance Assessment in net/ wall games. Teaching Elementary Physical Education, 14(2), 16-21.

Keating, X. D. (2003). The current often implemented fitness tests in physical education programs: problems and future directions. Quest, 55(2), 141-160.

Kuehl-Kitchen, J. M. (2005). Pre-service Teachers' experiences in planning, implementing and 
assessing the tactical (TGFU) model (The Florida State University). Tallahassee, Estados Unidos.

Light, R., \& Fawns, R. (2003). Knowing the game: integrating speech and action in games teaching trough TGfU. Quest, 55(2), 161-176.

López, V. M. (2011). La evaluación en Educación Física. Revisión de los modelos tradicionales y planteamiento de una alternativa: La evaluación formativa y compartida (2da ed.). Buenos Aires: Miño y Dávila.

López, V. M., Kirk, D., Lorente, E., MacPhail, A., \& Macdonald, D. (2013). Alternative assessment in physical education: a review of international literature. Sport, Education and Society, 18(1), 57-76.

MacPhail, A., Kirk, D., \& Griffin, L. L. (2008). Throwing and catching as relational skills in game play: Situated learning in a modified game unit. Journal of Teaching in Physical Education, 27(1), 100-115.

Mann, M. D. (2012). Systematic observation of coach feedback in elite youth volleyball (University of Arkansas). Fayetteville, Arkansas, Estados Unidos.

Memmert, D., \& Harvey, S. (2008). The Game Performance Assessment Instrument (GPAI): some concerns and solution. Journal of Teaching in Physical Education, 27(2), 220-221.

Méndez, A. (2011a). Coevaluación en los deportes de invasión desde el contexto de juego. En L. Martínez y R. Gómez (Eds.), La Educación Física y el Deporte en la Edad Escolar: el Giro Reflexivo en la Enseñanza (2da ed., pp. 327-347). Buenos Aires: Miño y Dávila.

Méndez, A. (2011b). La Evaluación desde la perspectiva comprensiva. Dificultades y estrategias didácticas para valorar el rendimiento de juego en situaciones modificadas. Tándem. Didáctica de la Educación Física, 37, 42-54.

Mesquita, I., Farias, C., \& Hastie, P. (2012). The impact of a hybrid Sport Education-Invasion Games Competence Model soccer unit on students' decision making, skill execution and overall game performance. European Physical Education Review, 18(2), 205-219.

Mesquita, I., Graça, A., Gomes, R., \& Cruz, C. (2005). Examinig the impact of a step game approach to teaching volleyball on student tactical decision making and skill execution during game play. Journal of Human Movement Studies, 48(6), 469-492.

Meztler, F. W. (2000). Tactical games: Teaching games for understanding. Boston, MA.: Allyn and Bacon.

Mitchell, S. A., \& Oslin, J. L. (1999). An investigation of tactical transfer in net games. European Journal of Physical Education, 4(2), 162-172.

Mitchell, S. A., Oslin, J. L., \& Griffin, L. L. (2006). Teaching sport concepts and skills: A tactical games approach (2da ed.). Champaign, IL: Human Kinetics.

Moher, D., Liberati, A., Tetzlaff, J., \& Altman, D. G. (2009). Preferred reporting items for systematic reviews and meta-analyses: The PRISMA statement. Annals of Internal Medicine, 151(4), 264269.

Moreno, A., Del Villar, F., García-González, F., Gil, A., \& Moreno, M. P. (2011). Intervención en la toma de decisiones en jugadores de voleibol en etapas de formación. Revista de Psicología del Deporte, 20(2), 785-800.

Moreno, M. P., Moreno, A., García-González, L., Gil, A., Claver, F., \& Del Villar, F. (2011). Elaboración de herramientas cognitivas para la detección, seguimiento y optimización de talentos deportivos en voleibol. Archivos de Medicina del Deporte, 28(146), 435-446.

Musch, E., Mertens, B., Timmers, E., Mertens, T., Graça, A., Taborsky, F., .. Vonderlynck, V. (2002). An innovative didactical invasion games model to teach basketball and handball. Presentado en Annual Congress of the European College of Sport Science, Atenas, Grecia.

Oslin, J. 1., Mitchell, S. A., \& Griffin, L. 1. (1998). The Game Performance Assessment Instrument (GPAI): development and preliminary validation. Journal of Teaching in Physical Education, 17(2), 231-243. 
Otero, F. M., González, J. A., \& Calvo, A. (2012). Validación de instrumentos para la medición del conocimiento declarativo y procedimental y la toma de decisiones en el fútbol escolar. Retos: nuevas tendencias en educación física, deporte y recreación, 22, 65-69.

Panton, S. A. (2006). The effects of a teacher induction program on graduate student teacher effectiveness in physical education (The Florida State University). Tallahassee, Estados Unidos.

Perlman, D., Forrest, G., \& Pearson, P. (2012). Nintendo Wii: Opportunities to put the education back into physical education. Australian Journal of Teacher Education, 37(7), 85-94.

Pritchard, T., Hawkins, A., \& Wiegand, R. (2008). Effects of two instructional approaches on skill development, knowledge, and game performance. Measurement in Physical Education and Exercise Science, 12(4), 219-236.

Psotta, R., \& Martin, A. (2011). Changes in decision making skill and skill execution in soccer performance: the intervention study. Acta Universitatis Palackianae Olomucensis, 41(2), 7-15.

Richard, J. F., \& Griffin, L. L. (2003). Authentic assessment in games education: an introduction to team sport assessment procedure and the game performance assessment instrument. En J. I. Butler, L. L. Griffin, B. Lombard, \& R. Natasi (Eds.), Teaching games for understanding. Physical education and sport (pp. 156-166). Reston, V.A.: AAHPERD Publications.

Roberts, S. (2007). Performance in invasion games: an assessment of information communication technology/post graduate certificate in education physical education teachers. Physical Education Matters, 2(3), 41-45.

Siedentop, D. (1994). Sport education: quality PE through positive sport experiences. Champaign: Human Kinetics Publishers.

Siendetop, D. (2002). Ecological perspectives in teaching research. Journal of Teaching in Physical Education, 21(4), 427-440.

Tallir, I., Lenoir, M., Valcke, M., \& Musch, E. (2007). Do Alternative instructional approaches result in different game performance learning outcomes? Authentic Assessment in varying game. International Journal of Sport Psychology, 38(3), 263-282.

Whipp, P., Jackson, B., Dimmock, J. A., \& Soh, J. (2015). The effects of formalized and trained non-reciprocal peer teaching on psychosocial, behavioural, pedagogical, and motor learning out comes in physical education. Frontiers in Psychology, 6(149), 1-13.

Wright, S., McNeill, M., Fry, J., \& Wang, J. (2005). Teaching teachers to play and teach games. Physical Education \& Sport Pedagogy, 10(1), 61-82. 
